\title{
Adaptive Observer for Parabolic PDEs with Uncertain Parameter in the Boundary Condition
}

\author{
Tarek Ahmed-Ali, Fouad Giri, Miroslav Krstic, Laurent Burlion, Françoise Lamnabhi-Lagarrigue
}

\begin{abstract}
We are considering the problem of state observation for a class of infinite dimensional systems. The latter are modelled by a parabolic PDE and a boundary condition involving an unknown parameter. The problem is dealt with using an adaptive observer that provides online estimates of the system (spatially distributed) state and the unknown parameters. The observer is formally shown to be exponentially convergent under an ad-hoc persistent excitation condition.
\end{abstract}

Index Terms - parabolic PDE, state observation, parameter estimation, adaptive observer.

\section{INTRODUCTION}

Practical implementation of control systems may necessitate, not only online measurements of the system outputs, but also those of the internal state variables. The point is that, in most situations, it is not possible to have full information on the system state variables, due to the fact that not all these variables are accessible to measurements or the costs of implementing all the necessary sensors are prohibitive. This is particularly the case when infinite dimensional systems are involved. In such situations, the only solution is to use state observers to get online estimates of all state variables. One of the most known state estimators is the Luenberger observer [1]. This established the structure that most observers are based upon today. Surveys on observer design can be found in [2,3], for finite-dimensional systems, and in [4] for infinite dimensional systems.

In real-life control problems, the online state estimation issue is usually faced in presence of large system parameter uncertainty. Then, the problem is addressed by the design of adaptive state observers which provide online estimates of both the states and the unknown parameters. The first adaptive observers have been developed for finite-dimensional continuous-time linear systems and an extensive overview is found in $[5,6]$. The case of (finitedimensional) nonlinear systems has been extensively studied leading to several solutions, e.g. [7-13].

The problem of observer design for infinite dimensional systems (IDSs) has also been given a great deal of interest. Several observer design techniques have been developed including the infinite dimensional Luenberger observer for linear IDSs (e.g. $[4,14,15,16])$, the boundary observer design of bilinear IDSs (e.g. $[17,18,19,27])$, backstepping-based boundary observers for

T. Ahmed-Ali and F. Giri are with the GREYC Lab, University of Caen Basse-Normandie, ENSICAEN, Caen, France.

M. Krstic is with the University of California at San Diego, La Jolla, CA 92093-0411, USA.

L. Burlion is with ONERA, the French Aerospace Lab, BP 74025, 31055 Toulouse, France.

F. Lamnabhi-Lagarrigue is with the LSS-CNRS, SUPELEC, EECI, 91192 Gif-sur-Yvette, France . parabolic partial integro-differential [19], initial state recovery algorithms for various linear and nonlinear IDSs [20-22].

In the last few years, much interest has been paid to simultaneous parameter and state estimation for IDSs, within various contexts. In [23], simultaneous state and parameter estimation has been introduced to deal with output-feedback adaptive control design for parabolic PDEs. In this context, the unknown parameters are tuned by gradient-type laws while the (spatially distributed) state is estimated using open-loop filters. The convergence of the estimates to their true values is not established. But, this is not required for the achievement of the control objectives. In [24], simultaneous state and parameter estimation has been performed to solve a parameter identification problem for reaction-advection type systems involving a single unknown parameter. Open- and closed-loop adaptive identifiers have been proposed where the unknown parameter is estimated using gradient-type estimators, while the (spatially distributed) state is estimated using open-loop filters. It is shown that the parameter estimate converges to its true values, by just using constant exciting inputs. In [25], simultaneous parameter and state estimation has been considered within the context of adaptive stabilization for a wave equation subject to a boundary harmonic disturbance linearly parameterized along a known set of functions. An adaptive observer estimating the system state and the (disturbance) unknown parameters is proposed and the estimation error system is shown to be asymptotically stable.

In this paper, the problem of parameter and state estimation is addressed for IDSs that are described by a parabolic PDE with a boundary condition involving an uncertain parameter. This class of systems has been well motivated in $[24,28]$ where the emphasis was made on the identification of the unknown parameter. Presently, the objective is to accurately estimate both the state of domain and parameter of the boundary condition. To this end, an adaptive observer is designed that provides online estimates of the state (along the domain) and the parameter. The observer design is inspired from adaptive observer designs of nonlinear ODE systems and PDE systems. Accordingly, the observer includes state and parameter estimators as well as a gain adaptation law. A formal analysis of the proposed adaptive observer is developed leading to conditions on the input signal and the design parameter. Interestingly, the input is allowed to be of any type but is must be persistently exciting. Under this condition, the state and parameter estimation errors are made exponentially vanishing.

The paper is organized as follows: the observation problem statement, including the class of IDSs, is described is Section 2; the adaptive observer design and analysis are dealt with Sections 3 and 4, respectively; a conclusion and a reference list end the paper. 


\section{Observation Problem Statement}

The system under study is described by a parabolic PDE of the form:

$u_{t}(x, t)=u_{x x}(x, t), \quad 0 \leq x \leq 1, t \geq 0$

with the boundary condition:

$$
u_{x}(0, t)=-q u(0, t)
$$

and:

$$
u(1, t)=U(t) \quad \text { (control input) }
$$

$y(t)=u(0, t) \quad($ system output $)$

where $q \in \mathbf{R}$ is a fixed unknown parameter. It is only assumed that $q<1$ for the system (1a-d) to be (BIBO) stable.

The goal is to generate accurate online estimates, $\hat{u}(x, t)$ and $\hat{q}(t)$, of the system state $u(x, t) \quad(0 \leq x \leq 1 ; t \geq 0)$ and the parameter $q$, based only on the output measurements $(U(t), y(t) ; t \geq 0)$. To achieve this objective, it is assumed that the state variable $u(x, t) \quad(0 \leq x \leq 1 ; t \geq 0)$ as well as the input derivative $\dot{U}(t)=u_{t}(1, t)$ are bounded

\section{ADAPTIVE OBSERVER DESIGN}

The system model (1a-c) suggests the following observer structure:

$\hat{u}_{t}(x, t)=\hat{u}_{x x}(x, t)+v(x, t)$

$\hat{u}_{x}(0, t)=-\hat{q} u(0, t)-L(\hat{u}(0, t)-y(t))$

$\hat{u}(1, t)=U(t)$

where $\hat{q}(t)$ is a parameter estimate, $v(x, t)$ is an additional correction term. A suitable choice of these quantities will be made on the basis of the subsequent analysis. First, introduce the following errors:

$\tilde{u}(x, t)=\hat{u}(x, t)-u(x, t)$ (state estimation error)

$\tilde{q}(t)=\hat{q}(t)-q \quad$ (parameter estimation error)

Then, subtracting equation (1a) to (3a), it follows that $\widetilde{u}(x, t)$ undergoes the following equation:

$\tilde{u}_{t}(x, t)=\widetilde{u}_{x x}(x, t)+v(x, t)$,

with the following boundary conditions:

$\widetilde{u}_{x}(0, t)=-\widetilde{q} u(0, t)-L \tilde{u}(0, t)$

$\widetilde{u}(1, t)=0$

using (1b-c) and (3b-c). Now, let us introduce the following coordinate change: $z(x, t)=\widetilde{u}(x, t)-\lambda(x, t) \widetilde{q}(t)$,

where $\lambda(x, t) \in \mathbf{R}$ is an auxiliary function to be defined later. Then, it follows from (6) that $z(x, t)$ undergoes the following equation:

$z_{t}(x, t)=\tilde{u}_{x x}(x, t)+v(x, t)-\lambda_{t}(x, t) \tilde{q}(t)-\lambda(x, t) \dot{\widetilde{q}}(t), t \geq 0$

using (5a). Equation (7) suggests the following choice of $v(x, t)$ :

$v(x, t)=\lambda(x, t) \dot{\widetilde{q}}(t)$

Doing so, equation (7) simplifies to:

$z_{t}(x, t)=\tilde{u}_{x x}(x, t)-\lambda_{t}(x, t) \tilde{q}(t), \quad t \geq 0$

In view of $(6), z(x, t)+\lambda(x, t) \tilde{q}(t)$ can be substituted to $\tilde{u}(x, t)$ on the right side of (9). Then, one gets:

$$
\begin{aligned}
& z_{t}(x, t)=z_{x x}(x, t)+\lambda_{x x}(x, t) \tilde{q}(t)-\lambda_{t}(x, t) \tilde{q}(t) \\
& \quad=z_{x x}(x, t)+\left(\lambda_{x x}(x, t)-\lambda_{t}(x, t)\right) \tilde{q}(t), \quad t \geq 0
\end{aligned}
$$

Equation (10) suggests the following trajectory for the auxiliary state vector $\lambda(x, t)$ :

$\lambda_{t}(x, t)=\lambda_{x x}(x, t)$

This is completed with the following boundary and initial conditions which will prove to be judicious:

$\lambda(1, t)=0, \lambda(x, 0)=0, \lambda_{x}(0, t)=-u(0, t)$

Doing so, equation (10) boils down to:

$z_{t}(x, t)=z_{x x}(x, t) ; t \geq 0$

In view of (11b) and (5b), one get from (6) the following boundary conditions:

$z(1, t)=\widetilde{u}(1, t)-\lambda(1, t) \widetilde{q}(t)=0$

$$
\begin{aligned}
z_{x}(0, t)= & \widetilde{u}_{x}(0, t)-\lambda_{x}(0, t) \widetilde{q}(t) \\
= & -\widetilde{q} u(0, t)-L \widetilde{u}(0, t)-\lambda_{x}(0, t) \widetilde{q}(t) \\
= & -\widetilde{q} u(0, t)-L(\widetilde{z}(0, t)+\lambda(0, t) \widetilde{q}(t))-\lambda_{x}(0, t) \widetilde{q}(t) \\
= & -L \widetilde{z}(0, t)-\widetilde{q}\left(u(0, t)+L \lambda(0, t)+\lambda_{x}(0, t)\right) \\
& =-L z(0, t)-\widetilde{q} L \lambda(0, t)
\end{aligned}
$$

where the last equality is obtained using the last part of (11b). Owing to the unknown parameter, the following adaptive law is proposed: 


$$
\begin{aligned}
& \dot{\hat{q}}(t)=-\rho_{1} r(t) \lambda(0, t) \widetilde{y}(t) \\
& \dot{r}(t)=\rho_{2}\left(r(t)-r^{2}(t) \lambda^{2}(0, t)\right) ; \quad r(t) \in \mathbf{R} \\
& \widetilde{y}(t)=\hat{u}(0, t)-y(t)=\widetilde{u}(0, t)
\end{aligned}
$$

where the gains $\rho_{1}>0$ and $\rho_{2}>0$ as well as the initial conditions $\hat{q}(0) \in \mathbf{R}$ and $r(0)>0$ are all arbitrarily chosen. The observer thus designed is constituted of equations (3a-c), (11a-b) and (13a-c). For convenience, these equations are recapitulated in the following table:

\section{Table 1. Adaptive observer}

$$
\begin{aligned}
& \text { State observer: } \\
& \hat{u}_{t}(x, t)=\hat{u}_{x x}(x, t)+\lambda(x, t) \dot{\hat{q}}(t) \\
& \hat{u}_{x}(0, t)=-\hat{q} u(0, t)-L(\hat{u}(0, t)-y(t)), \quad \hat{u}(1, t)=U(t)
\end{aligned}
$$

Parameter estimator:

$$
\begin{aligned}
& \lambda_{t}(x, t)=\lambda_{x x}(x, t) ; \lambda(x, t) \in \mathbf{R} \\
& \lambda(1, t)=0, \lambda(x, 0)=0, \text { and } \quad \lambda_{x}(0, t)=-u(0, t) \\
& \dot{\hat{q}}(t)=-\rho_{1} r(t) \lambda(0, t) \tilde{y}(t), \quad \tilde{y}(t)=\hat{u}(0, t)-y(t) \\
& \dot{r}(t)=\rho_{2}\left(r(t)-r^{2}(t) \lambda^{2}(0, t)\right) ; r(t) \in \mathbf{R} ; r(0)>0
\end{aligned}
$$

It is worth noticing that equations $(14 \mathrm{c}-\mathrm{d})$ are similar to the filter (62)-(64) in [24]. However, the parameter adaptive law (14e-f) is different from the adaptive law (61) in [24]. The main feature of the former, compared to the latter, is that it offers the possibility of tuning independently the dynamics of the state estimator (14a-b), using the design parameter $L$, and those of the parameter adaptive law (14e), which is tuned by the parameters $\rho_{1}, \rho_{2}$. In [24], the dynamics of the state estimator and the parameter law can not be independently tuned.

\section{ADAPTIVE OBSERVER ANALYSIS}

The observer analysis relies on the study of the error system:

$$
\begin{aligned}
& z_{t}(x, t)=z_{x x}(x, t) ; t \geq 0, \\
& \dot{\widetilde{q}}(t)=-\rho_{1} r(t) \lambda(0, t) \tilde{y}(t) \\
& \tilde{y}(t)=\widetilde{u}(0, t)=z(0, t)+\lambda(0, t) \widetilde{q}(t) \\
& z_{x}(0, t)=-L z(0, t)-L \widetilde{q} \lambda(0, t), \quad z(1, t)=0 \\
& \lambda_{t}(x, t)=\lambda_{x x}(x, t) \\
& \lambda(1, t)=0, \lambda(x, 0)=0, \lambda_{x}(0, t)=-u(0, t)
\end{aligned}
$$

Clearly, (15a) is a simple copy of (12a); (15b) is obtained from (13a) and (4b); (15c) is obtained from (13c) and (6); (15d) is obtained from (12b-c); (15e-f) are copies of (11a-b). Before embarking in the analysis of the system (15a-b), recall the following Wirtinger's inequalities [26] as these will be repeatedly used: $\int_{0}^{1} \varphi^{2}(x) d x \leq \frac{4}{\pi^{2}} \int_{0}^{1} \varphi_{x}^{2}(x) d x$ and $\max _{0 \leq x \leq 1} \varphi^{2}(x) \leq \int_{0}^{1} \varphi_{x}^{2}(x) d x$

whatever $\varphi \in H^{1}(0,1)$ such that $\varphi(0)=0$ or $\varphi(1)=0$, where $H^{1}(0,1)$ is the Sobolev space of absolutely continuous scalar functions $\varphi$ such that $\varphi_{x} \in L_{2}(0,1)$.

The stability analysis relies upon the following persistent excitation assumption:

$$
\exists \delta, \varepsilon_{0}, \varepsilon_{1}>0, \forall t>0: \varepsilon_{0}<\int_{t}^{t+\delta} \lambda^{2}(0, s) d s<\varepsilon_{1}
$$

It can be shown (see e.g. [6,7]) that, the time-varying scalar $r(t)$ (solution of (14f)) does exist, is positive and stays bounded away from 0 , provided $\lambda(0, t)$ is bounded and satisfies the PE (18). The boundedness of $\lambda(0, t)$ is formally established in the following proposition:

Proposition 1. The signal $\lambda(0, t)$ is bounded

Proof. see Appendix A.

In the latter, the notation,

$\lambda_{\max }=\sup _{t \geq 0} \mid \lambda(0, t \mid$.

is used. Proposition 1 and condition (18) ensure that, in turn the inverse $r^{-1}$ is bounded, is positive and stays bounded away from 0 . Specifically, there are two positive real numbers $(r, \bar{r})$, such that:

$r_{0} \leq r^{-1}(t) \leq r_{1}$, for all $t \geq 0$

with $r_{0}=\inf _{t \geq 0} r^{-1}(t)$ and $r_{1}=\sup _{t \geq 0} r^{-1}(t)$. In the sequel, condition (18) is supposed to be true, so that one can make use of (19) Then, the following Lyapunov function is defined:

$V(z, \tilde{\theta})=r^{-1} \widetilde{q}^{2}+\int_{0}^{1} z^{2}(x, t) d x$

Theorem 1. Consider the adaptive observer of Table 1 and let its design parameters be selected as follows:

$$
0<L<1, \quad \rho_{1}=\frac{L}{2} \text {, and } \rho_{2}<2 L
$$

When applied to the system (1a-b)-(2a-b) then, the adaptive observer is exponentially convergent in the sense that $\int_{0}^{1} \widetilde{u}^{2}(x, t) d x$ and $\widetilde{q}(t)$ both converge exponentially to zero, whatever the initial conditions $\widetilde{q}(0)$ and $(\widetilde{u}(x, 0), 0<x<1)$

Proof. From (20) one gets the following time-derivative:

$\dot{V}(z, \widetilde{\theta})=\widetilde{q}^{2}(t) \dot{r}^{-1}(t)+2 \widetilde{q}(t) r^{-1}(t) \dot{\widetilde{q}}(t)+\int_{0}^{1} z(x, t) z_{t}(x, t) d x$

Using (15b) and (14f), one immediately gets: 


$$
\begin{aligned}
\widetilde{q}^{2}(t) \dot{r}^{-1}(t)+ & 2 \widetilde{q}(t) r^{-1}(t) \dot{\tilde{q}}(t) \\
= & \rho_{2} \widetilde{q}^{2}(t)\left(-r^{-1}+\lambda^{2}(0, t)\right)-2 \rho_{1} \widetilde{q}(t) \lambda(0, t) \widetilde{y}(t) \\
= & -\rho_{2} \widetilde{q}^{2}(t) r^{-1}+\rho_{2} \widetilde{q}^{2}(t) \lambda^{2}(0, t) \\
& -2 \rho_{1} \widetilde{q}(t) \lambda(0, t)(z(0, t)+\lambda(0, t) \widetilde{q}(t)) \quad \text { using }(15 \mathrm{c}) \\
= & -\rho_{2} \widetilde{q}^{2}(t) r^{-1}+\left(\rho_{2}-2 \rho_{1}\right) \widetilde{q}^{2}(t) \lambda^{2}(0, t) \\
& -2 \rho_{1} \widetilde{q}(t) \lambda(0, t) z(0, t) \\
\leq & -\rho_{2} r_{0} \widetilde{q}^{2}(t)+\left(\rho_{2}-2 \rho_{1}\right) \widetilde{q}^{2}(t) \lambda^{2}(0, t) \\
& -2 \rho_{1} \widetilde{q}(t) \lambda(0, t) z(0, t)(\operatorname{using}(19))
\end{aligned}
$$

On the other hand, using (15a) the third term on the right side of (21) develops as follows:

$$
\begin{aligned}
\int_{0}^{1} z(x, t) z_{t}(x, t) d x & =\int_{0}^{1} z(x, t) z_{x x}(x, t) d x \\
& =\left[z(x, t) z_{x}(x, t)\right]_{0}^{1}-\int_{0}^{1} z_{x}^{2}(x, t) d x
\end{aligned}
$$

where the last equality is obtained using an integration by part. The above equality yields, successively:

$$
\begin{aligned}
\int_{0}^{1} z(x, t) z_{t} & \left.(x, t) d x=-z(0, t) z_{x}(0, t)-\int_{0}^{1} z_{x}^{2}(x, t) d x \quad \text { (using }(15 \mathrm{~d})\right) \\
= & z(0, t)(L \widetilde{z}(0, t)+L \widetilde{q}(t) \lambda(0, t))-\int_{0}^{1} z_{x}^{2}(x, t) d x \quad(\text { by }(15 \mathrm{~d})) \\
& =L z^{2}(0, t)+L \widetilde{q}(t) \lambda(0, t) z(0, t)-\int_{0}^{1} z_{x}^{2}(x, t) d x
\end{aligned}
$$

Using (22) and (23), equation (21) implies:

$$
\begin{aligned}
& \dot{V} \leq-\rho_{2} r_{0} \widetilde{q}^{2}(t)+\left(\rho_{2}-2 \rho_{1}\right) \widetilde{q}^{2}(t) \lambda^{2}(0, t)-2 \rho_{1} \widetilde{q}(t) \lambda(0, t) z(0, t) \\
& +L z^{2}(0, t)+L \widetilde{q}(t) \lambda(0, t) z(0, t)-\int_{0}^{1} z_{x}^{2}(x, t) d x \\
& \leq-\rho_{2} r_{0} \widetilde{q}^{2}(t)+\left(\rho_{2}-2 \rho_{1}\right) \widetilde{q}^{2}(t) \lambda^{2}(0, t) \\
& +L z^{2}(0, t)+\left(L-2 \rho_{1}\right) \widetilde{q}(t) \lambda(0, t) z(0, t)-\int_{0}^{1} z_{x}^{2}(x, t) d x \\
& \leq-\rho_{2} r_{0} \widetilde{q}^{2}(t)+\left(\rho_{2}-2 \rho_{1}\right) \widetilde{q}^{2}(t) \lambda^{2}(0, t) \\
& +L z^{2}(0, t)+\left(L-2 \rho_{1}\right) \tilde{q} \lambda(0, t) z(0, t)-\zeta \int_{0}^{1} z_{x}^{2}(x, t) d x \\
& -(1-\zeta) \int_{0}^{1} z_{x}^{2}(x, t) d x \quad(\text { whatever } 0<\zeta<1) \\
& \leq-\rho_{2} r_{0} \widetilde{q}^{2}(t)+\left(\rho_{2}-2 \rho_{1}\right) \widetilde{q}^{2}(t) \lambda^{2}(0, t) \\
& +(L-\zeta) z^{2}(0, t)+\left(L-2 \rho_{1}\right) \widetilde{q} \lambda(0, t) z(0, t) \\
& -(1-\zeta) \int_{0}^{1} z_{x}^{2}(x, t) d x \text { (using the second part in (16)) } \\
& \leq-\rho_{2} r_{0} \widetilde{q}^{2}(t)-(1-\zeta) \int_{0}^{1} z^{2}(x, t) d x \\
& -\left[\begin{array}{l}
\tilde{q} \lambda(0, t) \\
z(0, t)
\end{array}\right]^{T}\left[\begin{array}{cc}
\left(2 \rho_{1}-\rho_{2}\right) & -\frac{L-2 \rho_{1}}{2} \\
-\frac{L-2 \rho_{1}}{2} & -(L-\xi)
\end{array}\right]\left[\begin{array}{l}
\tilde{q} \lambda(0, t) \\
z(0, t)
\end{array}\right]
\end{aligned}
$$

where the last inequality is obtained using the first part in (16) The matrix in the last quantity of (23) is made positive semidefinite iff:

$2 \rho_{1}-\rho_{2}>0$ and $-(L-\zeta)\left(2 \rho_{1}-\rho_{2}\right)-\frac{\left(L-2 \rho_{1}\right)^{2}}{4} \geq 0$

It is readily checked that, the above inequalities hold if the following conditions are satisfied:

$L=2 \rho_{1}=\zeta$ and $\rho_{2}<2 \zeta$

where $0<\zeta<1$ is arbitrary. It turns out that, the adaptive observer parameters must be selected as follows:

$0<L<1, \quad \rho_{1}=\frac{L}{2}$, and $\rho_{2}<2 L$

Doing so, inequality (23) gives:

$\dot{V} \leq-\rho_{2} r_{0} \widetilde{q}^{2}(t)-(1-\zeta) \int_{0}^{1} z^{2}(x, t) d x$

which shows that $\dot{V}$ is negative definite which, in view of (20), implies that both $\tilde{q}(t)$ and $\int_{0}^{1} z^{2}(x, t) d x$ are exponentially convergent to zero.

Now, from (6) one has:

$\widetilde{u}(x, t)=z(x, t)+\lambda(x, t) \widetilde{q}(t)$

Since $\int_{0}^{1} \lambda^{2}(x, t) d x$ is bounded (by Proposition 1), it readily follows that, in turn, $\int_{0}^{1} \widetilde{u}^{2}(x, t) d x$ exponentially converges to zero. This completes the proof of Theorem 1

\section{CONCLUSION}

The problem of state observation and parameter estimation has been addressed of IDSs described by the model (1a-c)-(2a-b). The latter is basically a parabolic PDE with uncertainty in the boundary condition. The problem is coped with using the adaptive observer (14a-f) which is shown to enjoy the exponential convergence feature of Theorem 1 , in presence of the persistent excitation property (18).

\section{Appendix A. Proof of Proposition 1}

Part 1. Following a similar proof in [24], let us consider the transformation:

$e=u-q \lambda-\eta$ 
with $\eta$ defined by:

$$
\begin{aligned}
& \eta_{t}(x, t)=\eta_{x x}(x, t) \\
& \eta(1, t)=u(1, t), \text { and } \eta_{x}(0, t)=0
\end{aligned}
$$

It is easily checked that $e$ undergoes the following equations:

$$
\begin{aligned}
& e_{t}(x, t)=e_{x x}(x, t) \\
& e_{x}(0, t)=0, \text { and } e(1, t)=0
\end{aligned}
$$

It is shown in many places that the system (A4)-(A5) is exponentially stable (e.g. [29]). Then, it readily follows from (A1) that, $\lambda(0, t)$ is bounded if and only if $\eta(0, t)$ is so. To show this, consider the Lyapunov function candidate:

$W(t)=\frac{1}{2} \int_{0}^{1} \eta_{x}^{2}(x, t) d x$

Time-derivative of $W$, along the system (A2)-(A3), yields:

$$
\begin{aligned}
\dot{W}(t) & =\int_{0}^{1} \eta_{x}(x, t) \eta_{x t}(x, t) d x \\
& =\int_{0}^{1} \eta_{x}(x, t) \eta_{t x}(x, t) d x \text { (because } \eta \text { is smooth) } \\
& =\left[\eta_{x}(x, t) \eta_{t}(x, t)\right]_{0}^{1}-\int_{0}^{1} \eta_{x x}^{2}(x, t) d x \\
& =\eta_{x}(1, t) \eta_{t}(1, t)-\int_{0}^{1} \eta_{x x}^{2}(x, t) d x
\end{aligned}
$$

using successively an integration by part and the fact that $\eta_{x}(0, t)=0$. Furthermore, the first condition in (A3) gives $\eta_{t}(1, t)=u_{t}(1, t)$. Then, (A7) implies, using Young inequality:

$$
\begin{aligned}
\dot{W}(t) & =\frac{1}{2 \xi^{2}} u_{t}^{2}(1, t)+\frac{\xi^{2}}{2} \eta_{x}^{2}(1, t)-\int_{0}^{1} \eta_{x x}^{2}(x, t) d x \\
\leq & \frac{1}{2 \xi^{2}} u_{t}^{2}(1, t)+\frac{\xi^{2}}{2} \int_{0}^{1} \eta_{x x}^{2}(x, t) d x-\int_{0}^{1} \eta_{x x}^{2}(x, t) d x \\
\leq & \frac{1}{2 \xi^{2}} u_{t}^{2}(1, t)-\left(1-\frac{\xi^{2}}{2}\right) \int_{0}^{1} \eta_{x x}^{2}(x, t) d x
\end{aligned}
$$

whatever $0<\xi<\sqrt{2}$, where the second inequality is obtained using (the second part in) (16). Again, using the first inequality in (16), one gets from (A8):

$$
\begin{aligned}
\dot{W}(t) & \leq \frac{1}{2 \xi^{2}} u_{t}^{2}(1, t)-\left(1-\frac{\xi^{2}}{2}\right) \int_{0}^{1} \eta_{x}^{2}(x, t) d x \\
& \leq \frac{1}{2 \xi^{2}} u_{t}^{2}(1, t)-\left(1-\frac{\xi^{2}}{2}\right) W
\end{aligned}
$$

This implies that $\int_{0}^{1} \eta_{x}^{2}(x, t) d x$ is bounded, because $u_{t}(1, t)=\dot{U}(t)$ is bounded by assumption. Furthermore, one has:

$$
\begin{gathered}
|\eta(0, t)|=\left|\eta(1, t)-\int_{0}^{1} \eta_{x}(x, t) d x\right|=\left|u(1, t)-\int_{0}^{1} \eta_{x}(x, t) d x\right| \\
\leq|u(1, t)|+\left|\int_{0}^{1} \eta_{x}(x, t) d x\right| \\
\leq|u(1, t)|+\left(\int_{0}^{1} \eta_{x}^{2}(x, t) d x\right)^{1 / 2}
\end{gathered}
$$

where we have used Schwartz inequality to get the last inequality. Clearly, inequality (A10) shows that $|\eta(0, t)|$ is bounded. This establishes that $|\lambda(0, t)|$ is bounded and completes the proof of Proposition 1

\section{REFERENCES}

[1] Luenberger D. G. Observing the state of a linear system. IEEE T. on Mil. Electron., vol. 8, no. 2, pp. 74-80, Apr. 1964.

[2] Misawa E. A. and J. K. Hedrick. Nonlinear observers - A state-of-the art survey. J. Dyn. Syst.-T. ASME, vol. 111, no. 3, pp. 344-352, 1989.

[3] Spurgeon S. K. Sliding mode observers: A survey. Int. J. Syst. Sci., vol. 39, no. 8, pp. 751-764, 2008.

[4] Hidayat Z., R. Babuska, B. De Schutter, and A. Nunez. Observers for linear distributed-parameter systems: A survey. IEEE International Symposium on Robotic and Sensors Environments, Montreal, Canada, pp. 166-171, 2011.

[5] Narendra K.S. and A.M. Annaswamy (1989). Stable Adaptive Systems. Prentice Hall. Reprinted by Dover Publications, New York, 2004.

[6] Ioannou P. and J. Sun (1996). Robust Adaptive Control. Prentice Hall.

[7] G. Kreisselmeier, "The generation of adaptive law structures for globally convergent adaptive observers," IEEE Trans. Automat. Contr., vol. 24, no. 3, pp. 510-513, 1979.

[8] Bastin G., Gevers M.R. (1988). Stable adaptive observers for nonlinear time-varying systems. IEEE Transactions on Automatic Control; vol. 33, pp. 650-658.

[9] Marino R, Tomei P. (1996). Nonlinear control design: geometric, adaptive and robust. Prentice Hall, UK.

[10] Besançon G. (2000). Remarks on nonlinear adaptive observer design. Systems and Control Letters; vol. 41, pp.271-280.

[11] Zhang Q. (2002). Adaptive observer for multiple-inputmultiple-output (mimo) linear time-varying systems. IEEE Trans. Automatic Control, vol. 47 (3), pp. 525-529.

[12] Besançon G., J. De León-Morales, O. Huerta-Guevara (2006). On adaptive observers for state affine systems. International Journal of Control, vol. 79 (6), pp. 581-591.

[13] Besançon G., (2007). Nonlinear Observers and Applications, Springer, ISBN: 978-3-540-73502-1.

[14] Curtain R.F. and H. Zwart (1995). An Introduction to Infinite Dimensional Linear Systems Theory. Springer-Verlag, New York.

[15] Lasiecka I., R. Triggiani, (2000). Control Theory for Partial Differential Equations: Continuous and Approximation Theories, Cambridge University Press, Cambridge, UK. 
[16] Amann H. (1989), Feedback stabilization of linear and semilinear parabolic systems. In: Semigroup Theory and Applications, Clément et al. (editors). Lecture Notes Pure Appl. Math. 116, 21-57, M. Dekker, New York, 1989.

[17] Bounit H. and H. Hammouri, (1997). Observers for infinite dimensional bilinear systems. European J. Control, vol. 3 (1), pp. 325-339.

[18] Vries D., K.J. Keesman, H. Zwart (2007). A Luenberger observer for infinite dimensional bilinear system: a UV desinfection example. IFAC Symposium on System, Structure and Control, Foz do Iguaçu, Brazil.

[19] Smyshlyaev A., Krstic M., (2005). Backstepping observers for a class of parabolic PDEs. Systems \& Control Letters, vol. 54, pp. 613-725.

[20] Tucsnak, M., G. Weiss, (2009). Observation and control for operator semigroups. Basel, Birkhäuser.

[21] Ramdani K., M. Tucsnak, G. Weiss (2010). Recovering the initial state of an infinite-dimensional system using observers. Automatica, vol. 46, pp. 1616-1625.

[22] Fridman E. (2013). Observers and initial state recovering for a class of hyperbolic systems via Lyapunov method. Automatica, vol. 49, pp. 2250-2260.

[23] Smyshlyaev A. and M. Krstic (2006). Output-Feedback Adaptive Control for Parabolic PDEs with Spatially Varying Coefficients, 45th IEEE Conference on Decision \& Control, San Diego, USA.

[24] Smyshlyaev A., M. Krstic, Adaptive boundary control for unstable parabolic PDEs-Part III: Output feedback examples with swapping identifiers. Automatica, vol. 43, pp.1557-1564, 2007.

[25] Guo W. and B.Z. Guo, (2013). Parameter Estimation and Non-Collocated Adaptive Stabilization for a Wave Equation Subject to General Boundary Harmonic Disturbance. IEEE Transactions on Automatic Control, vol. 58(7), pp. 1631-1643.

[26] Hardy G., J. Littlewood, and G. Polya (1934). Inequalities. Cambridge: Cambridge University Press.

[27] Xu C.Z., P. Ligaius, and J.P. Gauthier, (1995). An observer for infinite-dimensional dissipative bilinear systems. Comput. Math. Appl., vol. 29 (7), pp.13-21.

[28] Smyshlyaev A., Y. Orlov, and M. Krstic (2009). Adaptive Identification of two unstable PDEs with boundary sensing and actuation. Int. J. Adaptive Control and Signal Processing, vol. 23, pp. 131-149.

[29] Smyshlyaev A. and M. Krstic. Adaptive Control of Parabolic PDEs. Princeton University Press, 2010. 